\title{
First real-time detection of solar pp neutrinos by Borexino
}

M. Pallavicini ${ }^{1,2, a}$, G. Bellini' ${ }^{3}$, J. Benziger ${ }^{4}$, D. Bick ${ }^{5}$, G. Bonfini ${ }^{6}$, D. Bravo ${ }^{7}$,

B. Caccianiga ${ }^{3}$, F. Calaprice ${ }^{8}$, A. Caminata ${ }^{1,2}$, P. Cavalcante ${ }^{6}$, A. Chavarria ${ }^{8}$, A. Chepurnov ${ }^{9}$,

D. D’ Angelo ${ }^{3}$, S. Davini ${ }^{10}$, A. Derbin ${ }^{11}$, A. Empl ${ }^{10}$, A. Etenko ${ }^{12}$, K. Fomenko ${ }^{13,6}$,

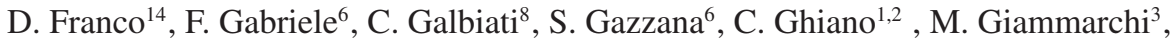

M. Göger-Neff ${ }^{15}$, A. Goretti ${ }^{8}$, M. Gromov ${ }^{9}$, C. Hagner ${ }^{5}$, E. Hungerford ${ }^{10}$, Al. Ianni ${ }^{6}$, An. Ianni ${ }^{8}$, M. Kayser ${ }^{5}$, V. Kobychev ${ }^{25}$, D. Korablëv ${ }^{13}$, G. Korga ${ }^{11}$, D. Kryn ${ }^{14}$,

M. Laubenstein ${ }^{6}$, B. Lehnert ${ }^{17}$, T. Lewke ${ }^{15}$, E. Litvinovich ${ }^{16}$, F. Lombardi ${ }^{6}$, P. Lombardi ${ }^{3}$,

L. Ludhova ${ }^{3}$, G. Lukyanchenko ${ }^{12}$, I. Machulin ${ }^{16}$, S. Manecki $^{7}$, W. Maneschg ${ }^{19}$,

S. Marcocci ${ }^{24}$, Q. Meindl ${ }^{5}$, E. Meroni ${ }^{3}$, M. Meyer ${ }^{5}$, L. Miramonti ${ }^{3}$, M. Misiaszek ${ }^{18}$,

M. Montuschi ${ }^{6}$, P. Mosteiro ${ }^{8}$, V. Muratova ${ }^{11}$, L. Oberauer ${ }^{15}$, M. Obolensky ${ }^{14}$, F. Ortica ${ }^{19}$,

K. Otis ${ }^{21}$, L. Papp ${ }^{7}$, L. Perasso ${ }^{2}$, A. Pocar ${ }^{21}$, G. Ranucci ${ }^{3}$, A. Razeto ${ }^{6}$, A. Re $^{3}$, A. Romani ${ }^{19}$,

N. Rossi ${ }^{6}$, R. Saldanha ${ }^{8}$, C. Salvo ${ }^{2}$, S. Schönert ${ }^{15}$, H. Simgen ${ }^{19}$, M. Skorokhvatov ${ }^{12,16}$,

O. Smirnov ${ }^{13}$, A. Sotnikov ${ }^{13}$, S. Sukhotin ${ }^{12}$, Y. Suvorov ${ }^{12,22}$, R. Tartaglia ${ }^{6}$, G. Testera ${ }^{2}$,

D. Vignaud ${ }^{14}$, R.B. Vogelaar ${ }^{7}$, F. von Feilitzsch ${ }^{15}$, H. Wang ${ }^{22}$, J. Winter ${ }^{15}$, M. Wojcik ${ }^{18}$,

M. Wurm ${ }^{23}$, O. Zaimidoroga ${ }^{13}$, S. Zavatarelli' ${ }^{2}$, K. Zuber ${ }^{17}$, and G. Zuzel ${ }^{18}$

${ }^{1}$ Università di Genova, Dipartimento di Fisica, via Dodecaneso 33, 16146 Genova, Italy

2 INFN Sezione di Genova, via Dodecaneso 33, 16146 Genova, Italy

${ }^{3}$ Dipartimento di Fisica, Università degli Studi and INFN, Milano 20133, Italy

${ }^{4}$ Chemical Engineering Department, Princeton University, Princeton, NJ 08544, USA

${ }^{5}$ Institut für Experimentalphysik, Universität Hamburg, Germany

${ }^{6}$ INFN Laboratori Nazionali del Gran Sasso, Assergi 67010, Italy

${ }^{7}$ Physics Department, Virginia Polytechnic Institute and State University, Blacksburg, VA 24061, USA

${ }^{8}$ Physics Department, Princeton University, Princeton, NJ 08544, USA

${ }^{9}$ Lomonosov Moscow State University Skobeltsyn Institute of Nuclear Physics, Moscow 119234,

Russia

${ }^{10}$ Department of Physics, University of Houston, Houston, TX 77204, USA

${ }^{11}$ St. Petersburg Nuclear Physics Institute, Gatchina 188350, Russia

${ }^{12}$ NRC Kurchatov Institute, Moscow 123182, Russia

${ }^{13}$ Joint Institute for Nuclear Research, Dubna 141980, Russia

${ }^{14}$ APC, Univ. Paris Diderot, CNRS/IN2P3, CEA/Irfu, Obs. de Paris, Sorbonne Paris Cité, France

${ }^{15}$ Physik Department, Technische Universität München, Garching 85747, Germany

${ }^{16}$ National Nuclear Research University "MEPhI”, 31 Kashirskoe shosse, Moscow, Russia

${ }^{17}$ Technische Universität Dresden, Institut für Kern und Teilchenphysik, Zellescher Weg 19, 01068 Dresden, Germany

${ }^{18}$ M. Smoluchowski Institute of Physics, Jagiellonian University, Crakow 30059, Poland

${ }^{19}$ Dipartimento di Chimica, Biologia e Biotecnologie, Università e INFN, Perugia 06123, Italy

${ }^{20}$ Max-Plank-Institut für Kernphysik, 69117 Heidelberg, Germany

${ }^{21}$ Physics Department, University of Massachusetts, Amherst, MA 01003, USA

${ }^{22}$ Physics ans Astronomy Department, University of California Los Angeles (UCLA), Los Angeles, CA 90095, USA

\footnotetext{
${ }^{a}$ e-mail: marco.pallavicini@ge.infn.it
}

(C) The Authors, published by EDP Sciences. This is an Open Access article distributed under the terms of the Creative Commons Attribution License 4.0 (http://creativecommons.org/licenses/by/4.0/). 
${ }^{23}$ Institut für Physik, Johannes Gutenberg Universität Mainz, Germany

${ }^{24}$ Gran Sasso Science Institute (INFN), 67100 L'Aquila, Italy

${ }^{25}$ Kiev Institute for Nuclear Research, Kiev 06380, Ukraine

Abstract. Solar neutrinos have been pivotal to the discovery of neutrino flavour oscillations and are a unique tool to probe the reactions that keep the Sun shine. Although most of solar neutrino components have been directly measured, the neutrinos emitted by the keystone pp reaction, in which two protons fuse to make a deuteron, have so far eluded direct detection. The Borexino experiment, an ultra-pure liquid scintillator detector running at the Laboratori Nazionali del Gran Sasso in Italy, has now filled the gap, providing the first direct real time measurement of pp neutrinos and of the solar neutrino luminosity.

\section{Introduction}

The study of low energy solar neutrinos (i.e. below 1-2 MeV, see Fig. 1) is relevant for two main reasons: on one hand, they offer a unique opportunity to investigate the behaviour of the Sun's interior and test the predictions of the Standard Solar Model; on the other, they allow to probe the MSW-LMA neutrino oscillation scenario in an energy range that is not accessible to water Cherenkov detectors $[1,2]$, which can detect solar neutrinos only above an energy threshold of about $3.5 \mathrm{MeV}$.

The original main goal of Borexino [3, 4] was the precise measurement of the rate induced by the monochromatic electron neutrinos $(0.862 \mathrm{keV})$ produced by the electron capture decay of ${ }^{7} \mathrm{Be}$ in the Sun $[5,6]$. However, the very high radio purity of the scintillator offered new unexpected results, such as a clear evidence of the pep solar neutrinos [7], a low energy threshold $(3 \mathrm{MeV})$ detection of ${ }^{8} \mathrm{~B}$ neutrinos [8], an unambiguous detection of geo-neutrinos [9], and several additional results on the search of rare events [10-13].

In this paper we report about an even less anticipated result, namely the real time detection of the solar neutrinos of lowest energy, the so called pp neutrinos, which were detected in the early 90's only by means of geochemical techniques [14], and its flux extracted indirectly. The Borexino direct measurement was made possible by the very low radioactive background, particularly in ${ }^{85} \mathrm{Kr}$, achieved after an extensive purification campaign performed in 20102011 and thanks to the extremely good performance of the detector as a whole.

The pp reaction $p p \longrightarrow d+e^{+}+v_{e}$ in the core of the Sun is the keystone process for energy production and is the source of the largest component of the neutrino flux. Its measurement is a major experimental milestone in solar neutrino physics, and paves the way to a deeper understanding of the Sun dynamics through a high precision comparison between photon and neutrino luminosity that future Borexino inspired detectors will be able to achieve.

Borexino is a liquid scintillator calorimeter [16-18] designed for the real-time observation of low energy solar neutrinos and anti-neutrinos. Neutrinos are detected through their elastic scattering on electrons and by means of the consequent emission of scintillation light, which is produced with a yield of about 500 p.e./MeV; anti-neutrinos are detected through inverse beta decay on protons. The total cross section of electron neutrinos depends on both charged and neutral currents weak interactions, while that of other neutrino flavours is induced by neutral currents only. The interaction rate depends therefore on the neutrino type at the target, a fact that makes the experiment sensitive to neutrino oscillations occurring along the path from the production site in the Sun's up to the detector.

The inner part of the Borexino detector [16] is an unsegmented stainless steel sphere (SSS) that is both the container of the scintillator and the mechanical support of the photomultipliers. Within this sphere, two nylon vessels separate the scintillator volume in 


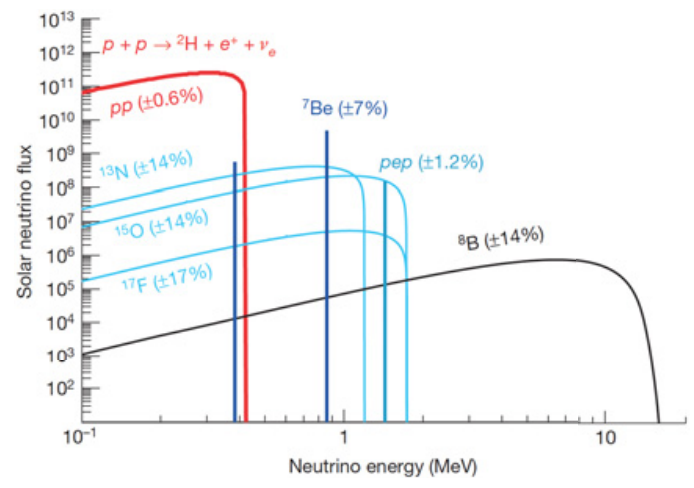

Figure 1. The spectrum of solar neutrinos as predicted by the Standard Solar Model [15].

three shells of radii $4.25 \mathrm{~m}, 5.50 \mathrm{~m}$ and $6.85 \mathrm{~m}$, the latter being the radius of the SSS itself. The inner nylon vessel (IV) contains the liquid scintillator solution (PC+PPO), while the second and the third shell contain PC with a small amount of light quencher in order to further reduce the scintillation yield of pure PC. The PC/PPO solution that we adopted as liquid scintillator guarantees high scintillation yield $\left(\approx 10^{4}\right.$ photons/MeV), high light transparency, and fast decay time $(\approx 3 \mathrm{~ns})$, all essential for good energy resolution, precise spatial reconstruction, and good discrimination between $\beta$-like events and events due $\alpha$ particles [19].

The scintillation light is collected by 2212 photomutipliers (PMTs) that are uniformly attached to the inner surface of the SSS. All but 384 PMTs are equipped with light concentrators that are designed to reject photons not coming from the active scintillator volume, thus reducing the background due to radioactive decays originating in the buffer liquid or $\gamma$ 's from the PMTs. The 384 PMTs without concentrators are used to study this background, and to help identify muons that cross the buffer, but not the Inner Vessel. Due to ageing, the actual number of operational PMTs during data taking was normally smaller, typically about 1800 in total.

The SSS is supported by 20 steel legs and enclosed within a large tank (WT) that is filled with ultra-pure water. The WT has a cylindrical base with a diameter of $18 \mathrm{~m}$ and a hemispherical top with a maximum height of $16.9 \mathrm{~m}$. The WT is a powerful shielding against external background ( $\gamma$ rays and neutrons from the rock) and is also used as a Cherenkov muon counter and muon tracker. The muon flux, although reduced by a factor of $10^{6}$ by the $3800 \mathrm{~m}$ w.e. depth of the Gran Sasso Laboratory, is of the order of $1 \mathrm{~m}^{-2} \mathrm{~h}^{-1}$, corresponding to about 4000 muons per day crossing the detector. A strong additional reduction factor (about $10^{4}$ ) is necessary to meet the Borexino requirements. Therefore the WT is equipped with 208 photomultipliers that collect the Cherenkov light emitted by muons in water. More details about the detector and its design principles can be found in [16-18].

The very high purity of the scintillator and the low radioactive background obtained in the innermost volume of the detector are the unique feature that has allowed all Borexino measurements and the one described in this paper in particular. The achievement of this good performance is the result of a long R\&D effort that is described elsewhere [3, 4, 20, 21].

Borexino data taking is split into two main periods: the so-called Phase-I, which lasted from 2007 until 2011, where we measured ${ }^{7} \mathrm{Be},{ }^{8} \mathrm{~B}$, pep and geo-neutrinos; the Phase-II, which has begun at the end of 2011 and which is still in progress. The pp-neutrinos analysis was done with Phase-II data only, being Phase-I data affected by a non-negligible ${ }^{85} \mathrm{Kr}$ background. In the next sections we describe the pp analysis and discuss the result. 

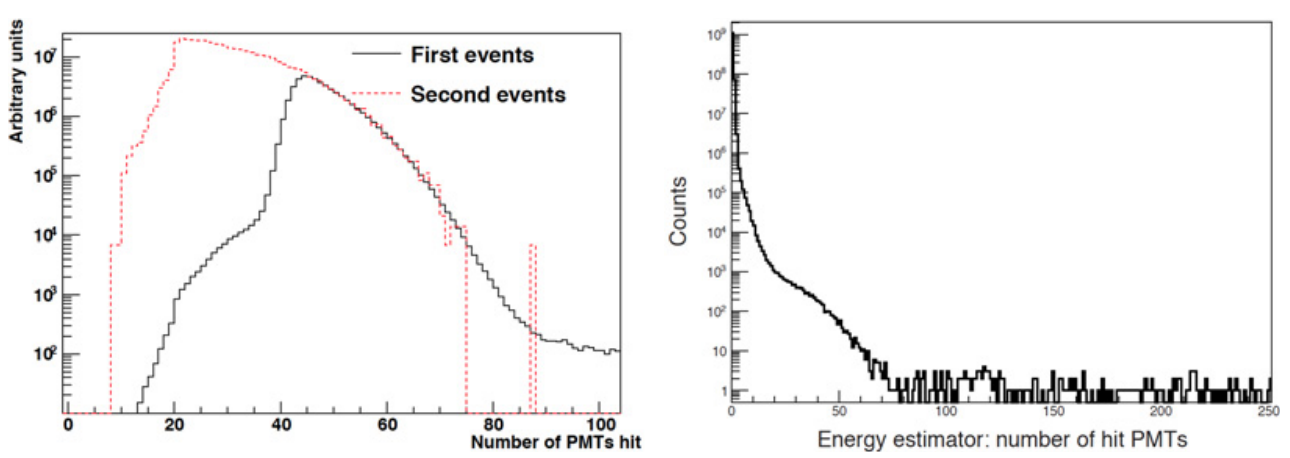

Figure 2. Left: spectra of first and second events in trigger windows acquired during the live time considered in the pp analysis. Second clusters are not subject to the effects of trigger threshold, and are thus useful for the independent estimate of the ${ }^{14} \mathrm{C}$ rate. Right: spectrum obtained by slicing random triggers into smaller time windows with the same duration as those used in regular data for the computation of our energy estimator.

\section{2. pp neutrino analysis}

The data used in this analysis was collected between Jan. 2012 and Jun. 2013, containing the first 408 live days of data of Borexino Phase-II. These data are characterised by reduced levels of some of the most relevant radiogenic backgrounds, particularly ${ }^{85} \mathrm{Kr},{ }^{210} \mathrm{Bi}$ and ${ }^{210} \mathrm{Po}$, thanks to a series of successful purification campaigns based on water extractions and low Argon-Krypton nitrogen sparging [23]. Basic selection criteria of this analysis are the same as previous Borexino solar neutrino papers and have been described in detail in [23], in the context of Borexino Phase-I. In particular, the criteria used in the pp analysis are:

1. No coincidence with muon events, identified both the the Cherenkov detector and with pulse-shape analysis in the scintillator;

2. Event triggered by a scintillation event in the inner detector;

3. No electronic noise from PMTs or electronics racks;

4. No coincidence with isotopic decays in the radon branch of the uranium series;

5. Position reconstruction within the fiducial volume; for this analysis, the fiducial volume is a truncated sphere with radius $\mathrm{R}=3.021 \mathrm{~m},|z|=1.67 \mathrm{~m}$; the fiducial volume is $86 \mathrm{~m}^{3}$, corresponding to 75.5 ton of scintillator target.

Most of the remaining background is ${ }^{14} \mathrm{C} \beta$-decays, whose end-point is $156 \mathrm{keV}$, very close to the pp region of interest. To estimate its rate independently from the main analysis, we looked at a sample of data in which the event causing the trigger is followed by a second event within the same trigger window (16 $\mu$ s long, while physical events last 100-500 ns). These second events are not subject to the trigger threshold (the typical threshold was 25 hits during data taking, corresponding to approximately $40 \mathrm{keV}$ in $\beta$-equivalent energy deposit), so they register down to much lower energies. The spectra of first and second events are shown in Fig. 2-left. By fitting this spectrum against the known ${ }^{14} \mathrm{C}$ spectral shape, we obtain a ${ }^{14} \mathrm{C}$ rate in Borexino of $40 \pm 1 \mathrm{~Bq}$ per $100 \mathrm{t}$. The error accounts for statistical and systematical uncertainties, most notably due to uncertainty in the ${ }^{14} \mathrm{C}$ spectral shape and fit conditions. Due to its high rate, ${ }^{14} \mathrm{C}$ naturally generates a considerable amount of pile-up: two physical events occur so close to each other in time that the detector fails to resolve them. With the above measurement of the ${ }^{14} \mathrm{C}$ rate, the expected rate of ${ }^{14} \mathrm{C}-{ }^{14} \mathrm{C}$ pile-up is 100 per day per $100 \mathrm{t}$. This is comparable to the expected pp neutrino interaction rate. In addition, the endpoint of the ${ }^{14} \mathrm{C}-{ }^{14} \mathrm{C}$ pile-up spectrum is $312 \mathrm{keV}$ (twice the ${ }^{14} \mathrm{C}$ spectrum end-point), also 
close to the endpoint of the pp-induced recoil spectrum, $\mathrm{E}_{R}^{\max }=264 \mathrm{keV}$. This underlines the importance of a careful assessment of pileup events, both ${ }^{14} \mathrm{C}-{ }^{14} \mathrm{C}$ and other pile-up.

The pile-up component can be determined using an independent, data-driven method which we called synthetic pileup. Real triggered events with no cuts are artificially overlapped with random data obtained from the ends of real trigger windows, uncorrelated with the triggering event. By overlapping four random data samples with each physical trigger, we obtain a sample of synthetic events that has four times the exposure of the real data set used. The synthetic events are then reconstructed using the same software used for real events, and selected with the same criteria. Using this method, we obtained the true rate and spectral shape of pile-up in our detector. This method naturally accounts for the effect of fiducialization on pile-up events.

With the ${ }^{14} \mathrm{C}$ and pile-up rates fixed, we obtained the pp rate by fitting the data spectrum in a window of $165-590 \mathrm{keV}$ against the known spectral shapes. The fit was performed with a tool named spectral-fitter, previously used in ${ }^{7} \mathrm{Be}$ [5] and pep [7] analyses. The tool was improved to work on any Borexino analysis. In particular, we included a treatment of the scintillator energy response to mono-energetic electrons at high statistics; a modified treatment of the energy resolution at low energy; and the introduction of synthetic pile-up. In addition to pp, ${ }^{14} \mathrm{C}$ and pile-up, other species included in the fit are other solar neutrinos $\left({ }^{7} \mathrm{Be}\right.$, CNO and pep), ${ }^{85} \mathrm{Kr},{ }^{210} \mathrm{Bi},{ }^{210} \mathrm{Po}$ and ${ }^{214} \mathrm{~Pb}$. The ${ }^{7} \mathrm{Be}$ rate was fixed to the rate previously obtained by Borexino [5]; $\mathrm{CNO}$ and pep rates were fixed to the values predicted by the highmetallicity Standard Solar Model; remaining background rates are left free in the fit, except for ${ }^{214} \mathrm{~Pb}$, which is fixed to the value measured by looking at ${ }^{214} \mathrm{Bi}^{214} \mathrm{Po}$ coincidences. The scintillator light yield and two energy resolution parameters are free to vary in the fit. The energy spectrum after cuts, together with the best fit, is shown on Fig. 3. The uncertainties shown in the figure are statistical only.

We considered also an alternative method to account for pile-up, which we called the convolution method. Random trigger with $16 \mu$ s-long samples of data are regularly collected by Borexino. These data are sliced into time windows of the same duration as the window used for our energy estimator. By computing the energy estimator inside each of these small windows, we generate the distribution shown in Fig. 2-right. This distribution is the spectrum collected by Borexino without a trigger threshold, and it includes electronic noise, as well as all physical events generated by signal and background species. Pile-up can be generated by convolving each spectral component (neutrinos and backgrounds) with this spectrum. Fit conditions were varied to estimate the systematic uncertainty. Effects considered include variations in: 1) The pile-up evaluation method (synthetic and convolution); 2) The length of the time window in which we count PMTs hit; 3) The definition of the fiducial volume; 4) The expected value of the CNO neutrino detection rate; 5) The fit start and end points.

Fits were performed using many different combinations of all these. The histogram of the rates obtained in all these conditions is shown in Fig. 4. The root mean square of the distribution is $9.8(d \cdot 100 t)^{-1}$. In addition, the resolution of the position reconstruction algorithm $(20 \mathrm{~cm}$; Sect. 2) induces a $2 \%$ uncertainty in the determination of the fiducial volume. These uncertainties are added in quadrature to obtain the total systematic uncertainty of our measurement. The final pp neutrino interaction rate is:

$$
R_{p p}=144 \pm 13(\text { stat }) \pm 10(\text { syst })(d \cdot 100 t)^{-1} .
$$

We also studied possible variations in the result caused by: 1) uncertainties in the spectral shapes of ${ }^{214} \mathrm{Po}$ and ${ }^{210} \mathrm{Bi}$; 2) variations of the expected pep rate within the measured and theoretical uncertainties; 3 ) leaving the ${ }^{214}$ Po rate free in the fit; 4) varying the veto time after muons cross the detector. No significant variations were observed. We also studied as a potential source of background the $\beta$-emitter ${ }^{87} \mathrm{Rb}$, with a Q-value of $283.3 \mathrm{keV}$, very 

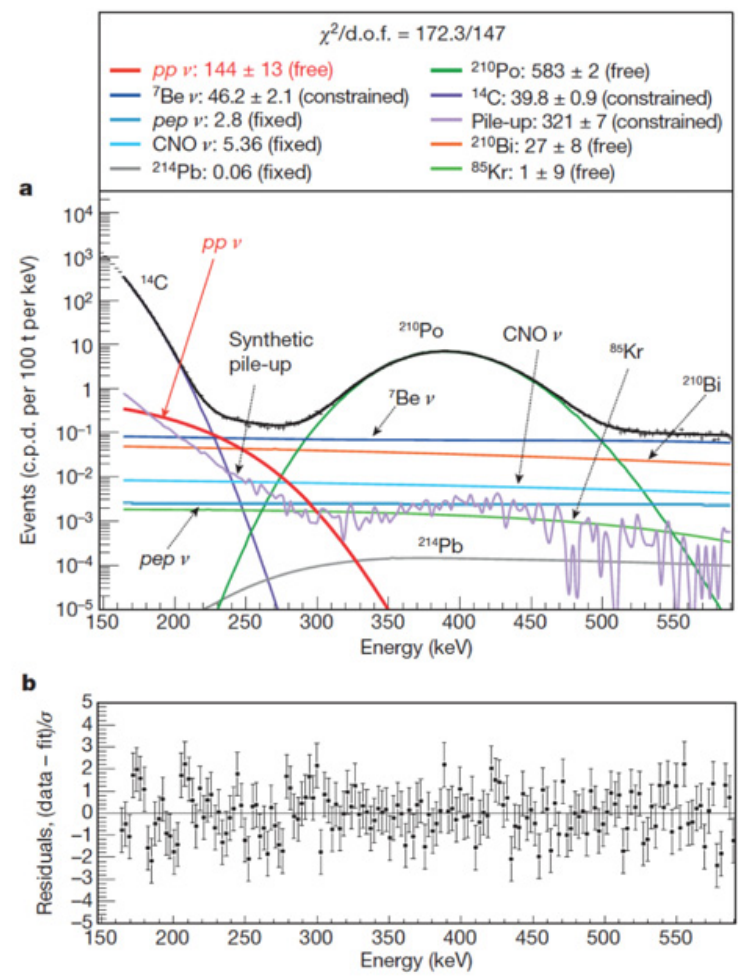

Figure 3. a) The fit result with all components explained within the figure. The fitted pp spectrum is shown in red; b) fit residuals as a function of the energy in the region of interest of pp neutrinos.

similar to the end-point of the pp-induced electron recoil spectrum $E_{R}^{\max }$. Using the known crustal abundances, isotopic abundances and half-lives of ${ }^{87} \mathrm{Rb}$ and ${ }^{40} \mathrm{~K}$, we can calculate the ratio between their natural activities to be 1 to 4 , respectively. Under the assumption that the relative abundances of ${ }^{87} \mathrm{Rb}$ and ${ }^{40} \mathrm{~K}$ in Borexino are equal to their relative crustal abundances, and knowing that the rate of ${ }^{40} \mathrm{~K}$ in Borexino is $<0.4(\mathrm{~d} \cdot 100 \mathrm{t})^{-1}$, we can constrain the ${ }^{87} \mathrm{Rb}$ rate to $<0.1(\mathrm{~d} \cdot 100 \mathrm{t})^{-1}$.

\section{Interpretation and conclusions}

The electron neutrino survival probability, $\mathrm{P}_{e e}$, is the probability for a solar neutrino produced as electron type to arrive at Earth as an electron-neutrino. It can be computed from the most up-to-date oscillation parameters and interaction cross sections, and the electron number density in Borexino, $(3.307 \pm 0.003) \times 10^{31}(100 \mathrm{t})^{-1}$ [6] (see [22] for details). Using that number and our measured pp neutrino interaction rate, we can calculate the pp neutrino flux to be $(6.6 \pm 0.7) \times 10^{10} \mathrm{~cm}^{-2} \mathrm{~s}^{-1}$, consistent with the SSM prediction. The uncertainty of the present measurement does not allow us to distinguish between the high and low-metallicity solar models.

Conversely, using pp neutrino flux predicted by the SSM as input, we calculate the survival probability $\mathrm{P}_{e e}=0.64 \pm 0.12$, consistent with expectations from the LMA-MSW solution [24], as shown in Fig. 5. Finally, the fact that the present measurement of the pp neutrino flux is consistent with expectation justifies the assumption made by the SSM that the photon and neutrino luminosities from the Sun can be correlated; i.e., that the Sun has 


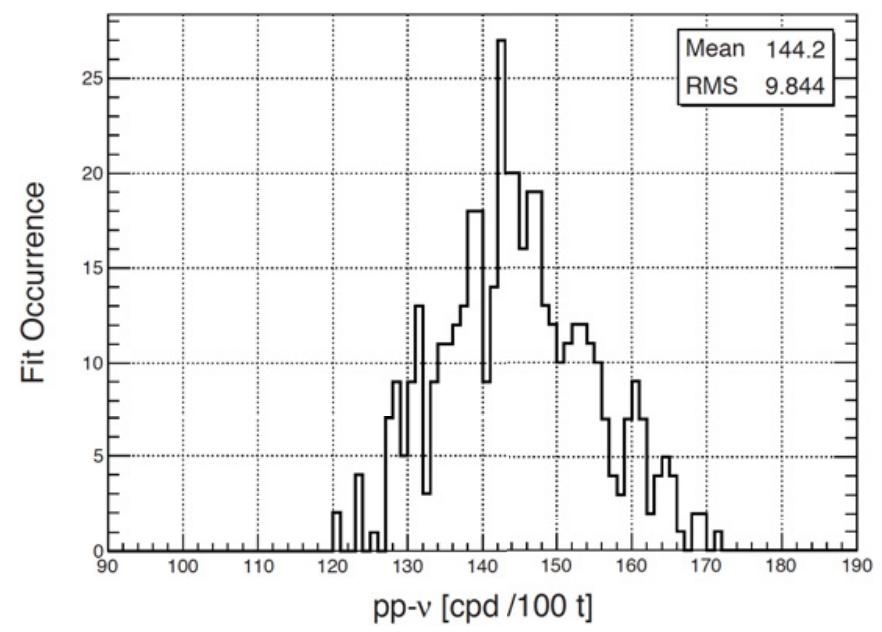

Figure 4. Distribution of the fit central value obtained by varying several fit conditions and parameters; the central value was taken as measurement central value; the sigma was used to estimate the systematic error to the measurement.

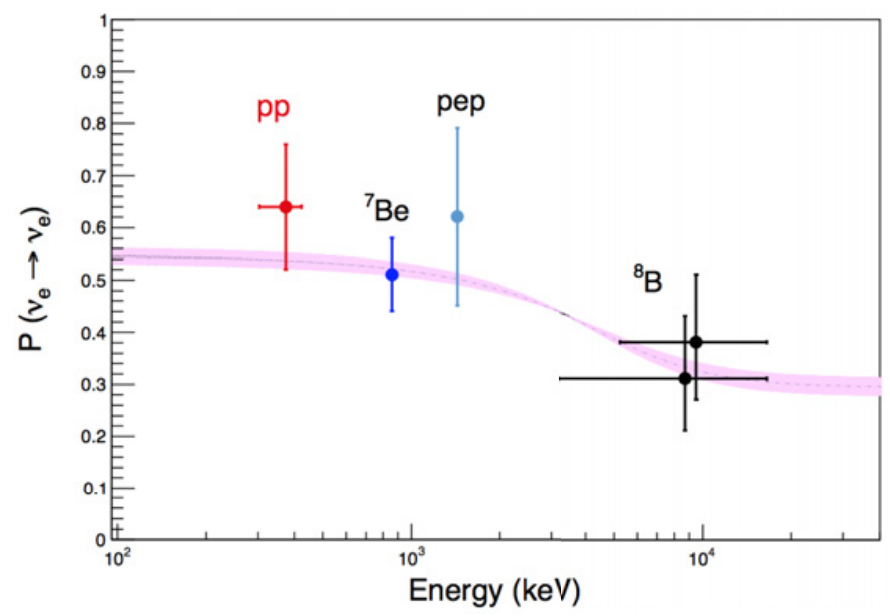

Figure 5. The contribution of Borexino to the experimental knowledge of the solar neutrino electron survival probability. In red, the measurement described in this work.

been stable for the past $10^{5}$ years. This invalidates a previous hypothesis that changes in the solar core over these time scales could be responsible for terrestrial global epochs [25].

In conclusion, the pp reaction in the Sun is the keystone process for energy production and is the source of the largest component of neutrino flux. The observation of the low-energy $(0-420 \mathrm{keV}) \mathrm{pp}$ neutrinos made by Borexino was possible because of the unprecedentedly low level of radioactivity reached inside the detector. The measured value is in good agreement with the predictions of both the high-metallicity and the low-metallicity SSMs. Although the experimental uncertainty does not yet allow the details of these models to be distinguished, this measurement confirms our understanding of the Sun. Future Borexinoinspired experiments might be able to measure solar pp neutrinos with the level of precision $(\approx 1 \%$ or better) needed to cross-compare photon and neutrino solar luminosities, while 
providing insight into solar dynamics over $10^{5}$-yr timescales. At the same time, such a precise measurement of pp neutrinos would yield the ultimate test for the MSW-LMA neutrino oscillation model and allow precision tests for exotic neutrino properties.

The Borexino experiment has measured all neutrino components related to the pp cycle. An attempt to measure CNO neutrinos, and therefore contribute to the solution of the metallicity problem, will be done in the next years, possibly with the help of additional purification cycles. The Borexino detector will also be used to search for sterile neutrinos by means of artificial neutrino and anti-neutrinos sources in the framework of the SOX project [26].

The Borexino program is made possible by fundings from INFN (Italy); NSF (USA); BMBF, DFG and MPG (Germany); JINR, RFBR, RSC and NRC Kurchatov Institute (Russia); and NCN (Poland). We acknowledge the generous support of the Laboratori Nazionali del Gran Sasso (Italy) and that of the Gran Sasso Science Institute. SOX is funded by ERC with grant ERC-AdG-2012 N. 320873.

\section{References}

[1] K. Abe et al. (Super-Kamiokande Collaboration), Phys. Rev. D 83 (2011) 052010

[2] B. Aharmin et al. (SNO collaboration), Phys. Rev. C 81 (2010) 055504

[3] G. Alimonti et al. (Borexino Collaboration), Astropart. Phys. 16 (2002) 205

[4] C. Arpesella et al. (Borexino Collaboration), Astropart. Phys. 18 (2002) 1

[5] G. Bellini et al. (Borexino Collaboration), Phys. Rev. Lett. 107 (2011) 141302

[6] G. Bellini et al. (Borexino Collaboration), Phys. Lett. B 707 (2012) 22-26

[7] G. Bellini et al. (Borexino collaboration), Phys. Rev. Lett. 108 (2012) 051302

[8] G. Bellini et al. (Borexino collaboration), Phys. Rev. D 82 (2012) 033006

[9] G. Bellini et al. (Borexino collaboration), Phys. Lett. B 722 (2013) 295-300

[10] G. Bellini et al. (Borexino collaboration), JCAP 1308 (2013) 049

[11] G. Bellini et al. (Borexino collaboration), Phys. Rev. D85 (2012) 092003

[12] G. Bellini et al. (Borexino collaboration), JINST 6 (2011) P05005

[13] G. Bellini et al. (Borexino collaboration), Phys. Lett. B696 (2011) 191-196

[14] W. Hampel et al. (Gallex Collaboration), Phys. Lett. B447 (1999) 127-133

[15] A.M. Serenelli, W.C. Haxton, W. C and C. Penã-Garay, Astrophys. J. 743 (2011) 24

[16] G. Alimonti et al. (Borexino collaboration), Nucl. Instr. and Meth. A 600 (2009) 568

[17] G. Bellini et al. (Borexino collaboration), JINST 6 (2011) P05005

[18] G. Bellini et al. (Borexino collaboration), Nucl. Instr. and Meth. A 609 (2009) 58-78

[19] H.O. Back et al. (Borexino collaboration), Nucl. Instr. and Meth. A 584 (2008) 98-113

[20] H.O. Back et al. (Borexino collaboration), Phys. Lett. B 563 (2003) 23-34

[21] H.O. Back et al. (Borexino collaboration), Phys. Lett. B 525 (2002) 29-40

[22] G. Bellini et al. (Borexino Collaboration), Nature, 512 (2014) 383

[23] G. Bellini, et al. (Borexino Collaboration), Phys. Rev. D 89 (2014) 112007

[24] J. N. Bahcall, P. I. Krastev, A. Y., Smirnov, Phys. Rev. D 58 (1998) 096016

[25] G. A. Cowan, W. C. Haxton, Los Alamos Science 3 (2) (1982) 46

[26] G. Bellini et al. (Borexino Collaboration), JHEP 08 (2013) 038 